\title{
Phytoprotection
}

\section{Index des auteurs, volume 84}

Author Index, Volume 84

Volume 84, numéro 3, décembre 2003

URI : https://id.erudit.org/iderudit/008495ar

DOI : https://doi.org/10.7202/008495ar

Aller au sommaire du numéro

Éditeur(s)

Société de protection des plantes du Québec (SPPQ)

ISSN

0031-9511 (imprimé)

1710-1603 (numérique)

Découvrir la revue

Citer ce document

(2003). Index des auteurs, volume 84 : author Index, Volume 84.

Phytoprotection, 84(3), 181-182. https://doi.org/10.7202/008495ar d'utilisation que vous pouvez consulter en ligne.

https://apropos.erudit.org/fr/usagers/politique-dutilisation/ 


\section{Index des auteurs, volume 84 Author Index, Volume 84}

\section{A-B}

Allaire, $\mathrm{M}$

Antoun, $\mathrm{H}$.

Audy, P.

Aupinel, $P$.

Bacon, R.

Baker, L.

Barbier, Y.

Bauce, É.

Bélair, G.

Bélanger, A.

Bellerose, S.

Belzile, F.

Benoit, D.-L.

Bernier, L.

Berthiaume, R.

Boiteau, G.

Boivin, G.

Borges, A.A.

Borges-Pérez, A.

Bostanian, N. J.

Boulanger, R.

Bourdages, J.V.

Bourgeois, G.

Bournoville, R.

Brodeur, J.

Brouillette, $\mathrm{P}$.

Bussières, G.

\section{C-F}

Carisse, $\mathrm{O}$.

Carré, $\mathrm{S}$

Carrière, $\mathrm{Y}$.

Charles, M.T.

Choquette, D.

Chouinard, G.

Coderre, D.

Colignon, $\mathrm{P}$.

Comeau, A.

Cormier, D.

Cortesero, A.M.

Couture, L.

Daigle, N.

Dangi, O.P.

Danho, M.
Dauphinais, $\mathrm{N}$.

de Oliveira, D.

174, 177

171

Demianyk, C.J.

19

167

Désilets, $\mathrm{H}$.

179

9

Desrosiers, R.

165

177

155

Dionne, J.

Epardaud, M.

9

Fenster, A. 165

Fernández-Falcón, M. 149

85

168

115

170

169

176

167

85

133

115

149

149

1,105

179

169

175

9

Firlej, A.

93

Fleury, D.

171

Fournier, Y.

168

Francis, F.

121,141

\section{G-L}

Gaspar, C.

141

Gauvin, M.-J. 115

Goubault, M.

Gravel, V. 171

Grimaud E. $\quad 9$

Hamelin, R.C. 167

Haubruge, É. 59, 121, 141

Hébert, C. 85

Hogue, R. 178

Hulasare, R.B. $\quad 19$

69, 177 Ivany, J.A. 27

169 Jayas, D.S.

170 Jobin, T. 172

Krespi, L. $\quad 77$

Laflamme, G. 170

168, 172 Landré, $B$. 9

9 Lasnier, J. 1

69 Laverdière, L. 178

179 Le Floch, C. 167

175 Le, V.-Q. 173

93, 105, $170 \quad$ Leclerc, D. 178

93, 105 Lemay, R. 176

121, 141 Levasseur, C. 173

176 Lucas, É. 105

93,170

\section{M-P}

176

178

168

MacDonald, T. 172

MacQuarrie, C.J.K. 133

Marceau, J. 177

Marchand, S. $\quad 169$ 
Martinez, C.

Mecteau, M.

Mentag, R.

Mignon, J.

Morin, Y.

Nébié, $R$.

Nénon, J.-P.

Neumann, $P$.

Noël, A.

Otoidobiga, L.C.

Paré, J.

Pelletier, F.

Pelletier, M.-L.

Philion, V.

Plantegenest, M.

Plouffe, D.

Poinsot, D.

Pouleur, S.

Provost, C.

\section{R-Y}

Rancourt, B.

Rasmont, $\mathrm{P}$.

Rioux, D.

Rioux, S.
Rochefort, S. $\quad 69$

Sabbahi, R. $\quad 177$

Sanderson, J.B. $\quad 27$

Séguin, A. $\quad 173,178$

Shetlar, D.J. $\quad 69$

Simard, L. $\quad 177$

Simard, M. 178

Somerville, T. $\quad 167$

Stewart, R.K. $\quad 174$

Talbot, B.G. $\quad 168$

Terzo, M. $\quad 39$

Toussaint, V. 172

Tremblay, J.-S. 178

Trudeau, M. 1

Tweddell, R.J. 171, 179

Urbanova, K. $\quad 39$

Valterova, I. $\quad 39$

Vincent, C. 171, 174

White, N.D.G. $\quad 19$

Yao, M.K. $\quad 179$ 